\title{
Distal Femoral Pin Tracker Placement Prevents Pin Tract-induced Fracture in Robotic-Assisted Total Knee Arthroplasty
}

\author{
ji-Hoon Baek, MD $\quad$ Su Chan Lee, MD ${ }^{1}$ jin-Hong Kim, MD ${ }^{1}$ Hye Sun Ahn, MS ${ }^{1}$ Chang Hyun Nam, MD \\ ${ }^{1}$ Joint and Arthritis Research, Himchan Hospital, Seoul, Republic of \\ Korea \\ J Knee Surg 2023;36:435-438. \\ Address for correspondence Chang Hyun Nam, MD, Joint and \\ Arthritis Research, Department of Orthopaedic Surgery, Himchan \\ Hospital, 120, Sinmok-ro, Yangcheon-gu, Seoul 07999, Republic of \\ Korea (e-mail: himchanhospital@gmail.com).
}

\begin{abstract}
Keywords

- total knee arthroplasty

- robotic-assisted system

- complications

The purpose of this study was to determine the effectiveness of the placement of pin trackers in the medial sagittal plane of the distal femur in robotic-assisted total knee arthroplasty (TKA) over a minimum follow-up period of 3 months. From August 2020 to October 2020, a consecutive series of 81 TKAs were performed in 59 patients using the Triathlon posterior-stabilized total knee prosthesis with a robotic-assisted system (Mako) at our hospital. Patient charts were reviewed for complications associated with the pin sites, including fracture, infection, thigh pain, and the need for reoperation. No patients had any minor or major complications associated with distal femoral pins. This technique, which used pin trackers in the medial sagittal plane of the distal femur, could be a useful option for surgeons performing robotic-assisted TKA. This is a Level IV study.
\end{abstract}

Robotic-assisted surgical techniques have been shown to be effective in total knee arthroplasty (TKA) and result in more accurate placement of the femoral and tibial components. ${ }^{1,2}$ However, this approach is also associated with unique potential complications. ${ }^{3}$ One of the possible adverse events that can occur is pin tract-induced femoral periprosthetic fracture, which requires additional surgical management and hospitalization.

The first case of postoperative fracture was described in 2006 by Ossendorf et $\mathrm{al}^{4}{ }^{4}$ and femur fractures occurring along the pin holes used for the trackers have been documented in computer-assisted navigation pins in TKA patients. $^{3,5-8}$ The pin holes left behind following removal of the tracking pins are a type of bone defect, which represent a potentially relevant cause of bone stress that may increase the future risk of fracture. ${ }^{6,9}$ The fracture incidence at these femoral pin tracker sites has been reported to range from 1 to $1.4 \%$, ${ }^{6,7}$ so this complication is not particularly rare.

We hypothesized that the placement of pin trackers in the medial sagittal plane of the distal femur prevents possible

received

January 6, 2021

accepted after revision

July 22, 2021

article published online

September 10, 2021

complications associated with this technique in roboticassisted TKA. However, few studies have specifically addressed the placement of pin trackers in the distal femur in robotic-assisted TKA.

The purpose of this study was to determine the effectiveness of the placement of pin trackers in the medial sagittal plane of the distal femur in robotic-assisted TKA over a minimum follow-up period of 3 months.

\section{Materials and Methods}

The design and protocol of this retrospective study were approved by the Institutional Review Board of our hospital, which waived the requirement for informed consent.

From August 2020 to October 2020, a consecutive series of 81 TKAs was performed in 59 patients using the Triathlon (Stryker Orthopaedics, Mahwah, NJ, USA) posterior-stabilized total knee prosthesis with a robotic-assisted system (Mako) at our hospital. All primary robotic-assisted TKAs performed during the study period were included. Patient charts were

(c) 2021. Thieme. All rights reserved. Thieme Medical Publishers, Inc., 333 Seventh Avenue, 18th Floor, New York, NY 10001, USA
DOI https://doi.org/ 10.1055/s-0041-1735462. ISSN 1538-8506. 
reviewed to identify any complications associated with the pin sites, including fracture, infection, thigh pain, and the need for reoperation. The mean age at the time of TKA was 69.9 years (range, 57-83 years), and the study population included eight males and 51 females. The primary diagnosis before initial TKA was osteoarthritis in all patients. The mean follow-up period was 3.5 months (range, 3-4 months).

All surgical procedures were performed by a single surgeon using the standard medial parapatellar approach, with sacrifice of the anterior and posterior cruciate ligaments in all patients. No patellas were replaced; only osteophytes were excised in all patients. All implants were inserted using cement. Patients were mobilized immediately with weightbearing as tolerated, and active exercises began under the supervision of a physiotherapist. The patients underwent clinical and radiographic follow-up examinations at 2 weeks, 6 weeks, 3 months, 6 months, 9 months, 12 months, and 1 year postoperatively thereafter. During these follow-up evaluations, any patients who did not return for their scheduled visits were contacted by telephone. Two nurses and one private doctor identified and visited nonresponders.

\section{Surgical Technique}

For the tibial pins, two stab incisions were made over the medial tibial diaphysis. After fixing the tibial pins bicortically in the tibial diaphysis, the patient's knee joint was positioned at approximately 130 degrees flexion (-Fig. 1A). The distal and medial femoral area were exposed using the retractor tools (-Fig. 1B). The first femoral pin was placed at a level approximately $1.5 \mathrm{~cm}$ superior and $1.5 \mathrm{~cm}$ posterior to the most prominent point of the medial epicondyle through the pin guide (-Fig. 2). The first pin was placed from medial to lateral approximately 15 degrees in the proximal direction ( - Fig. 3A) in order not to interfere with the box cutting chisel on the prepared distal femur during surgery (-Fig. 3B). The second pin entered the femoral distal shaft centrally (-Fig. 2). Both pins were inserted medial to the vastus medialis. Finally, the pins were centrally located on the sagittal femoral shaft and at 15-degree proximal angulation, sufficient to avoid making femoral notch cut in an anteroposterior position. All pins were placed unicortically with a self-drilling pin on power, and the pins were advanced until they were fully seated in the bone. Tracking arrays were then oriented medially ( - Fig. 4). Following completion of surgery, the pins and tracking arrays were removed.

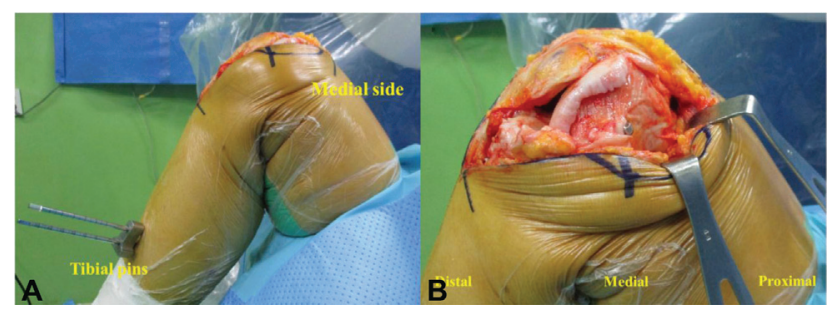

Fig. 1 (A) Tibial pins were placed approximately $20 \mathrm{~cm}$ inferior to the tibial joint line in the diaphysis. The patient's knee joint was positioned at approximately 130 degrees flexion. (B) The distal and medial femoral area was exposed using Army-Navy retractors.

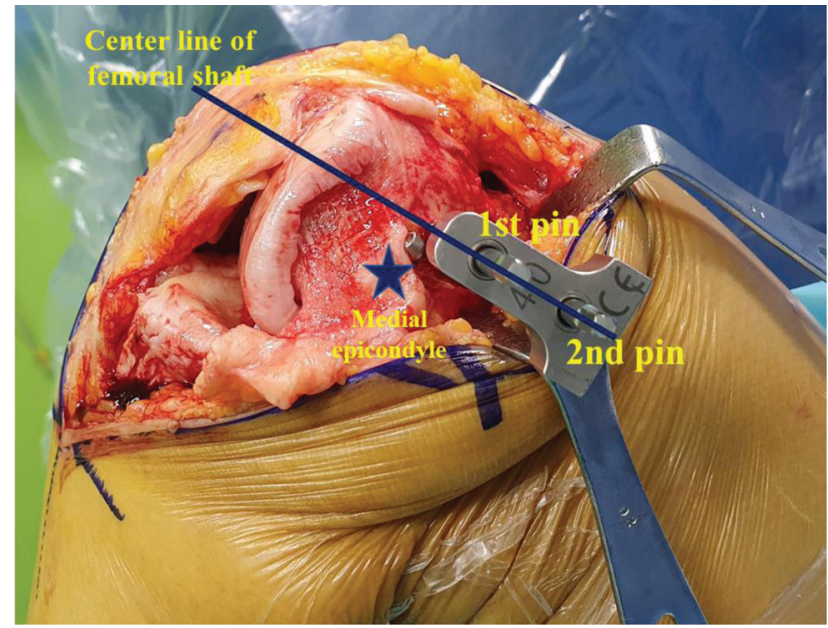

Fig. 2 A medial view of the femoral tracker pins. These pins are located within the incision. The first femoral pin was placed at a level approximately $1.5 \mathrm{~cm}$ superior and $1.5 \mathrm{~cm}$ posterior to the medial epicondyle, while the second pin entered the femoral distal shaft centrally.

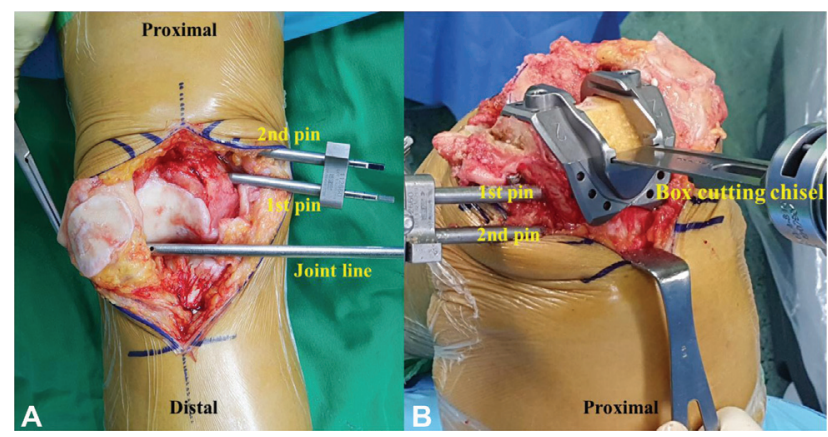

Fig. 3 (A) The first pin was placed from medial to lateral approximately 15 degrees in the proximal direction. (B) Note the position of the first pin placement and that the femoral notch cut is not affected by this pin placement.

\section{Results}

The study included 59 patients who underwent 81 total TKAs. No periprosthetic fractures at a pin tracker site were reported. In addition, no patients reported minor or major complications associated with the distal femoral pins.

\section{Discussion}

An increase in the incidence of pin site fractures was noted starting in 2006. ${ }^{4}$ These fracture reports implicate bicortical, unicortical, or transcortical placement of pins as the cause of fractures in the femoral shaft. ${ }^{3,5-8}$ Temporary femoral pins must be placed either within the operative field of the distal femur or percutaneously using separate stab incisions into the femoral shaft. However, little information is available concerning the placement of pin trackers in the distal femur during robotic-assisted TKA (- Table 1). Our technique uses unicortical placement of pin trackers in the medial sagittal plane of the distal femur. Since adopting this approach, we 


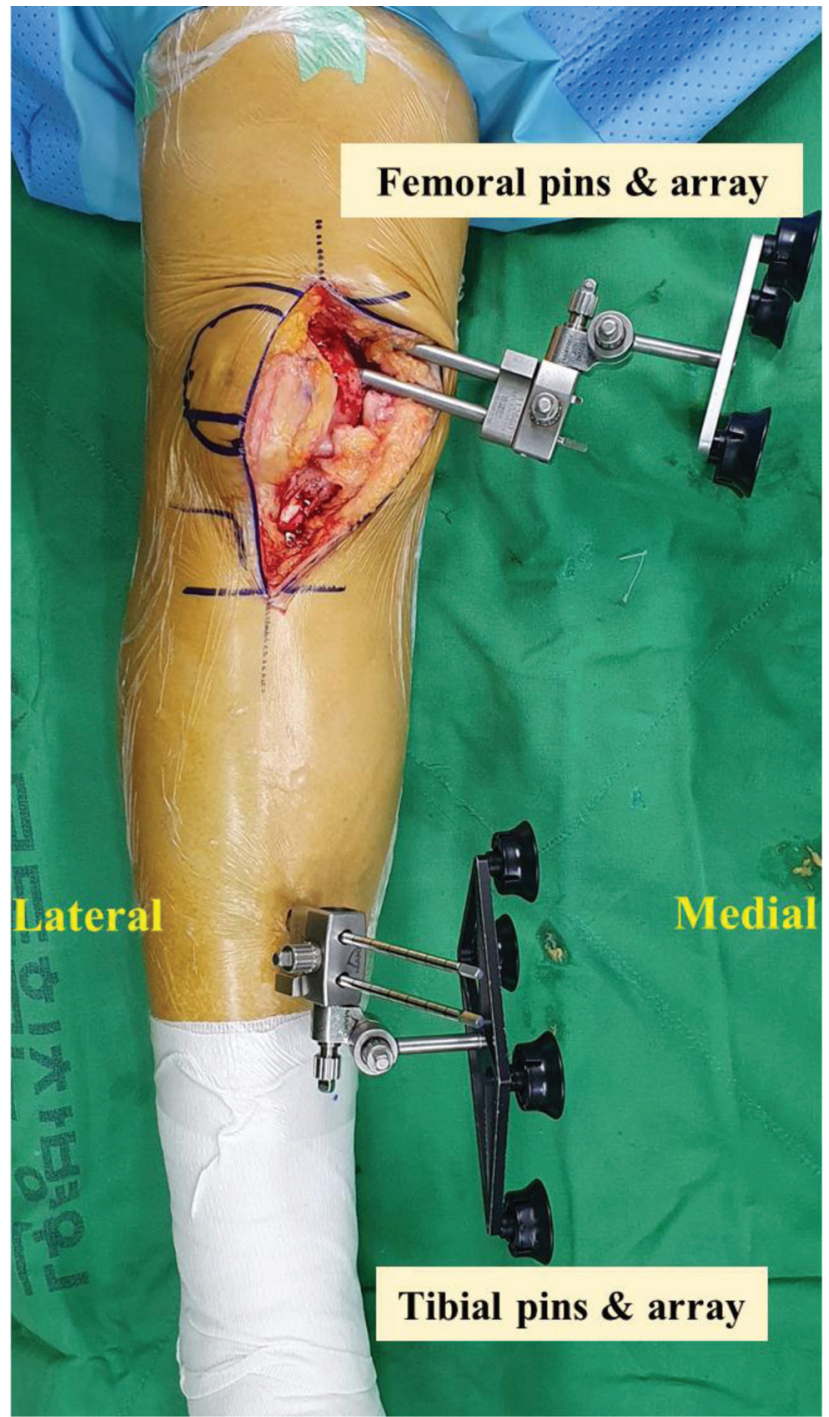

Fig. 4 An anterior view of the femoral and tibial tracker pins and a reference array.

have not experienced any fractures, neurovascular injuries, or pin site infections.

Complications related to these pin sites have been documented in adult patients undergoing TKA with computer- assisted navigation pins. ${ }^{6,7}$ The potential complications associated with pin trackers include pin site infection, neurovascular injury, soft-tissue morbidity, and fracture. ${ }^{3}$ Among these complications, fracture is serious, because it requires readmission to the hospital and additional surgical management, which both have significant associated costs and morbidity. ${ }^{8}$ Beldame et $\mathrm{al}^{6}$ reported the incidence of fractures at femoral pin tracker sites to be $1.4 \%$ in a series of 385 TKAs, while Wysocki et al ${ }^{7}$ identified a rate of approximately $1 \%$ in 210 TKAs. Therefore, patients should be informed preoperatively about this complication because it is not rare.

Distal femoral tracker pin placement has several advantages. First and foremost, because the surgeon directly fixes the tracker pins to the distal femoral bone in the medial to a lateral direction ( $\mathbf{F i g . 2}$ ), this procedure makes it possible to avoid soft-tissue and neurovascular injuries and therefore to correctly perform fixation without iatrogenic issues. Second, we expect the fracture incidence noted at femoral pin tracker sites to decrease considerably with the adoption of our technique. The femur mainly undergoes mechanical stress during torsion and flexion, and this type of mechanical stress makes it more sensitive to cortical bone loss of the femoral shaft than bone loss in the distal femoral bone. ${ }^{6,10}$ Third, because we only register the front of the distal femur and the femoral cartilage surface during the initial virtual model build up step, femoral pin placement in the medial surface does not interfere with femoral checkpoints. Finally, as the femoral pins are located in the medial area of the distal femur, they can act as medial retractors during roboticassisted TKA. However, this technique may require a relatively larger incision for patients with stiff soft tissue or obesity.

This study had several inevitable limitations. First, the study was retrospectively designed and only included a small number of patients. We also did not have a control group to compare participants who did not undergo distal femoral tracker pin placement. However, the study could not be prospectively randomized because fractures associated with femoral pin tracker sites are not rare. ${ }^{6,7}$ The followup period was also short. While a short follow-up period might not be enough to confirm long-term complications or guarantee the prevention of fractures associated with pin

Table 1 Reports of previous research into femoral pin tracker site fractures

\begin{tabular}{|c|c|c|c|c|}
\hline Author(s) (year) & $\begin{array}{l}\text { Number of } \\
\text { cases }\end{array}$ & $\begin{array}{l}\text { Femoral fracture } \\
\text { location }\end{array}$ & $\begin{array}{l}\text { Time from TKA to } \\
\text { fracture (weeks) }\end{array}$ & Other \\
\hline Ossendorf et al ${ }^{4}$ & 1 & $\begin{array}{l}1 \text { femoral shaft } \\
\text { (stress fracture) }\end{array}$ & 12 & Case report \\
\hline Jung et $\mathrm{al}^{5}$ & 1 & 1 femoral shaft & 6 & Case report \\
\hline Bonutti et al $\left.\right|^{3}$ & 2 & 2 femoral shaft & 912 & Case report \\
\hline Wysocki et $\mathrm{al}^{7}$ & 2 & 2 femoral shaft & 109 & Case report \\
\hline Beldame et al $^{6}$ & 5 (385 TKAs) & 5 femoral shaft & Average 12.6 & Case series \\
\hline Blue et $\mathrm{al}^{8}$ & 1 & 1 femoral shaft & 6 & Case report \\
\hline Present study & 0 (81 TKAs) & No & No & $\begin{array}{l}\text { Retrospective } \\
\text { observational study }\end{array}$ \\
\hline
\end{tabular}

Abbreviation: TKA, total knee arthroplasty. 
tracker sites during long-term follow-up, most of these reported complications have historically occurred within or around the first 3 postoperative months. ${ }^{3-7,11}$

Despite these limitations, this study demonstrated that our technique of pin placement in the distal femur in roboticassisted TKA is safe and reliable. Therefore, this technique could be a useful option for surgeons who perform TKA using a robotic-assisted system.

\section{Conflict of Interest}

None declared.

\section{References}

1 Sires JD, Craik JD, Wilson CJ. Accuracy of bone resection in MAKO total knee robotic-assisted surgery. J Knee Surg 2019;34(07):745-748

2 Werner SD, Stonestreet M, Jacofsky DJ. Makoplasty and the accuracy and efficacy of robotic-assisted arthroplasty. Surg Technol Int 2014;24:302-306

3 Bonutti P, Dethmers D, Stiehl JB. Case report: femoral shaft fracture resulting from femoral tracker placement in navigated TKA. Clin Orthop Relat Res 2008;466(06):1499-1502
4 Ossendorf C, Fuchs B, Koch P. Femoral stress fracture after computer navigated total knee arthroplasty. Knee 2006;13(05): 397-399

5 Jung HJ, Jung YB, Song KS, Park SJ, Lee JS. Fractures associated with computer-navigated total knee arthroplasty. A report of two cases. J Bone Joint Surg Am 2007;89(10):2280-2284

6 Beldame J, Boisrenoult P, Beaufils P. Pin track induced fractures around computer-assisted TKA. Orthop Traumatol Surg Res 2010; 96(03):249-255

7 Wysocki RW, Sheinkop MB, Virkus WW, Della Valle CJ. Femoral fracture through a previous pin site after computer-assisted total knee arthroplasty. J Arthroplasty 2008;23(03):462-465

8 Blue M, Douthit C, Dennison J, Caroom C, Jenkins M. Periprosthetic fracture through a unicortical tracking pin site after computer navigated total knee replacement. Case Rep Orthop 2018;2018:2381406

9 Kim K, Kim YH, Park WM, Rhyu KH. Stress concentration near pin holes associated with fracture risk after computer navigated total knee arthroplasty. Comput Aided Surg 2010;15(4-6):98-103

10 Aaron RK, Scott R. Supracondylar fracture of the femur after total knee arthroplasty. Clin Orthop Relat Res 1987;(219):136-139

11 Jung KA, Lee SC, Ahn NK, Song MB, Nam CH, Shon OJ. Delayed femoral fracture through a tracker pin site after navigated total knee arthroplasty. J Arthroplasty 2011;26(03):505.e9-505.e11 